\section{Genes evolving}

\author{
T. Cavalier-Smith
}

Four Billion Years: An Essay on the Evolution of Genes and Organisms. By William F. Loomis. Sinauer: 1988. Pp. 286. Hbk $\$ 39.95, £ 29.95$; pbk $\$ 22.95$, £17.50. *

UNTIL we know how genes make daisies rather than dinosaurs, the present gulf between molecular and organismic evolutionists will remain. Any book on the evolution of genes and organisms is therefore bound to be somewhat disjointed.

Loomis's readable essay is much less coherent than it need be, however. The reason is that the author - like so many biochemists - seems rather ignorant of the structure of organisms; in particular, there is here all too little appreciation of the structural diversity of unicellular organisms, for which we are much closer to understanding the molecular basis of body form than we are for animals and plants, and therefore also their organismic evolution. The book is even weaker on phylogeny, which is scarcely discussed at all - a curious omission for an essay on the main steps in the evolution of life. Too few molecular biologists realize that without an accurate phylogeny one cannot even correctly identify these steps, let alone discuss them intelligently. Nor, without a detailed knowledge of the actual structure, development and life history of the specific types of organism actually involved in a particular step, can we realistically apply what little we yet know of molecular epigenetics to explaining how major new types of organism evolve.

Loomis is also shaky on mechanisms of evolution, failing to appreciate the central role of reproductive competition. This is seen not only in remarks such as "all selection is for survival" and "fertility is seldom a limiting factor", but also in the brevity of his reference to arguments invoking the role of 'selfish' DNA in genomic evolution. Given the neglect of both phylogeny and the idea of intragenomic spreading of DNA sequences, it is not surprising that he unquestioningly accepts the over-popular primordial gene fusion theory (rather than the transposon insertion theory) of the evolutionary origin of introns.

Most surprising, though, is the treatment of eukaryote cell evolution, which goes counter to the recently emerging consensus. Even though Loomis accepts the symbiotic origin of both chloroplasts and mitochondria, he thinks that the eukaryote (host) cell itself evolved from a photosynthetic cyanobacterium. He is unaware of the existence of eukaryotes without mitochondria, and their probable

* In Britain distributed until now by Blackwell Scientific, but from November 1988 by W.H. Freeman. evolutionary significance, and of the extensive writings of evolutionary protistologists over the past dozen years or so. Equally idiosyncratic is the overriding importance given to rising oxygen levels for everything from the 'loss' of bacterial introns to the extinction of trilobites and dinosaurs, and even the origin of placental mammals, birds, antelopes and woolly mammoths.

The discussion of the molecular evolution of animal form is interesting and sensible as far as pattern formation is concerned. But it is weak in its neglect of the physical basis of morphogenesis, and in its phylogenetic and morphological misconceptions. Loomis, it seems, believes that sponges are a mere colony of flagellates.

Apart from the account of the genetics of pattern formation, the book's main strength lies in the way the author illustrates its pervasive theme of gene divergence from ancestral genes by the use of aligned motifs from actual protein sequences. The conservation of gene sequences and the importance of both gene duplication and the formation of new chimaeric genes (a process I would call gene chimaeration) are made very clear. This, however, is very much a book by a biochemist. It is thin on cell and organismic evolution, abysmally so when it comes to plants, fungi and protozoa, to which little more than a couple of pages are allocated. The discussion of prebiotic and metabolic chemistry is pretty conventional.

Loomis says of his book that "The primary goal is to connect the experimental and conceptual facts into a coherent story rather than to analyse the strengths and weaknesses of each step along the way". But to whom is this story directed? Not to the 'intelligent layman', for there is too much biochemistry for that. Nor to the professional evolutionist - the author makes no claims to be presenting either new ideas or a critical analysis. The problem with not examining the strengths and weaknesses of the steps along the way is that the inexpert reader has no way of knowing which parts are firmly based (for example the symbiotic origin of chloroplasts) and which (for example the assumption that the first cells were fermenters rather than photosynthesizers) are based on little more than fashion. This is more a personal travelogue than a traditional work of science: one may learn as much if not more about the author's background, interests and limitations as about the places visited.

I echo Loomis's hope that his book will encourage others to attempt a broad synthesis of molecular and organismic approaches to the history of life on our planet. But let them do their homework and learn more about organisms and phylogeny, more about the actual fossil record, and more about evolutionary mechanisms before putting pen to paper. We need much more than 'thermodynamics and biophysics' to constrain us 'from wild speculation'. Although Four Billion Years contains much more molecular detail than Lucretius, in its quasi-linear approach it seems closer to him and to eighteenth-century transformationists in intellectual flavour than to modern postdarwinian evolutionary biology.

T. Cavalier-Smith is in the Department of Biophysics, Cell and Molecular Biology, King's College London, 26-29 Drury Lane, London $W C 2 B$ SRL, UK.

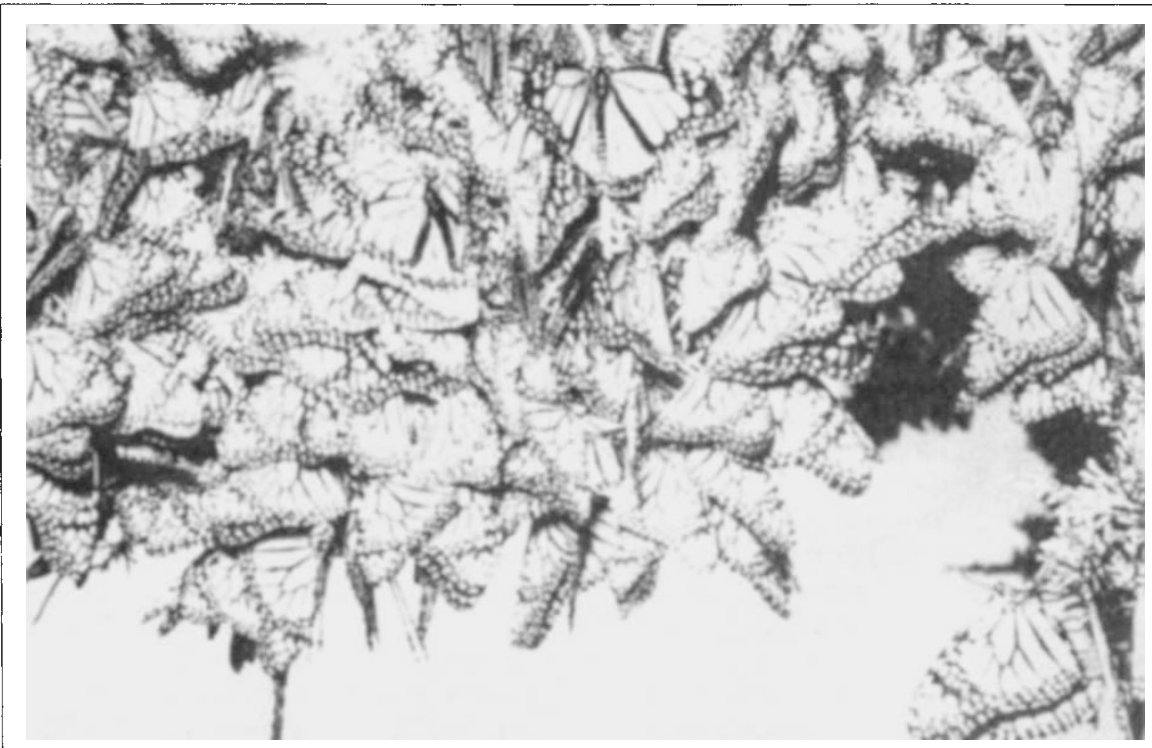

All a-flutter - a cluster of monarch butterflies overwintering in coastal California. The photograph ( $b y$ E. S. Ross) is taken from the fourth edition of Animal Behavior: An Evolutionary Approach by John Alcock. This edition has been largely rewritten: "the most important change

is my attempt to emphasize the tentative, unfinished nature of science and the significance of using multiple working hypotheses". Publisher is Sinauer, price is $\$ 34.95,124.95$ (for the British distributor see note opposite). 